\title{
Is x-ray compulsory in pubic symphysis diastasis diagnosis?
}

Sir

We read with great interest your case of postpartum symphysis pubis separation (1). We would like to present a case of pubic symphysis diastasis that we diagnosed with a different approach.

A 36-year-old gravida 1 para 0 at 39.6 weeks' gestation was admitted with the onset of spontaneous contraction. After three hours and 18 min she delivered a $3170 \mathrm{~g}$ baby without complications.

Three hours after delivery, she complained of severe pain in the symphysis pubic region. On examination, there was local tenderness in that region. We performed an ultrasound examination, which revealed a $15.2-\mathrm{mm}$ gap in the region of the symphysis pubis (Figure 1), diagnosed as pubic symphysis diastasis. She was given analgesics and advised bed rest. The patient was discharged six days after delivery and advised to maintain active ambulation and start physiotherapy. Three months later she was seen at the outpatient clinic. She was able to walk independently and was no longer experiencing any pain.

Although the case presented is not particularly impressive in severity, it still offers the opportunity to inform all clinicians about the possibility of diagnosing this condition with the use of the ultrasonography alone. The reported incidence of pubic symphysis diastasis varies widely in the literature, from 1 in 300 to 1 in 30000 deliveries $(2,3)$. Generally, it is a rare complication and for this reason it is very difficult to perform randomized controlled trials to compare different diagnostic tools. The diagnosis is based primarily on clinical findings. The most consistent finding is pain in the symphyseal region that radiates to the lower back and thighs and is exacerbated by leg movement (4). In addition, many women will have difficulty walking, in fact the gait is described as waddling, or potentially be unable to stand or walk due to pain (3). Symptoms may be noted dur-

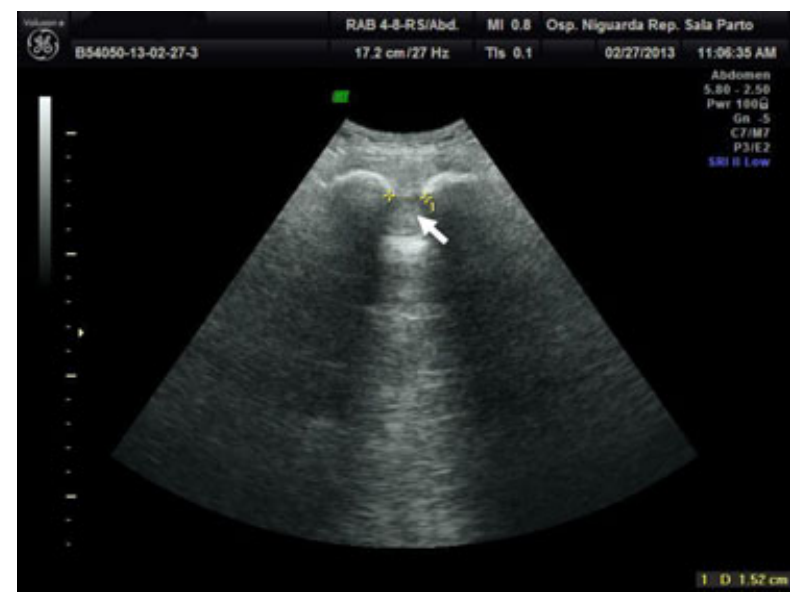

Figure 1. Ultrasound image showing pubic symphysis diastasis of $15.2 \mathrm{~mm}$ (arrow). ing labor and up to $48 \mathrm{~h}$ postpartum. Often the first diagnostic test used to identify the pubic diastasis is antero-posterior radiography. However, we think that ultrasound might be a good choice as an initial imaging study, rather than $\mathrm{x}$-ray, due to absence of exposure to ionizing radiation and its ease of operation, and as it presents an optimal assessment of the extent of symphysis separation (3-5). We performed ultrasonography in the following way: we placed the probe in transverse orientation on the pubic symphysis (identified by palpation) with an approximately $30^{\circ}$ caudal scanning plane, with the purpose of measuring the width of the symphyseal joint at its upper margin.

Pubic symphysis diastasis is an uncommon injury that should be considered when evaluating patients in the peripartum period who are experiencing suprapubic, sacroiliac or thigh pain. In addition we would like to bring to the general attention the usefulness of ultrasound in the diagnosis and management of this rare condition. The literature is inconsistent on this topic, due to the lack of randomized controlled trials, but good suggestions are present (35). In our experience, ultrasound is simple, reproducible and without side effects, and should be used as an initial imaging study because the accuracy is at least as good as that of $\mathrm{x}$-rays for estimating the width of the symphysis pubis diastasis.

Alessandro Svelato $^{1, *}$, Antonio Ragusa ${ }^{2}$, Antonino Perino ${ }^{1}$ and Mario Giuseppe Meroni ${ }^{2}$

${ }^{1}$ Department of Obstetrics and Gynecology, University Hospital Paolo Giaccone, Palermo, and ${ }^{2}$ Department of Obstetrics and Gynecology, Niguarda Hospital, Milan, Italy

*Corresponding Author: Alessandro Svelato

E-mail: alessandrosvelato@virgilio.it

DOI: $10.1111 /$ aogs.12275

\section{References}

1. Fidan U, Ulubay M, Keskin U, Frratlıgil FB, Karasahin KE, Ege T, et al. Postpartum symphysis pubis separation. Acta Obstet Gynecol Scand. 2013;92:1336-7.

2. Parker JM, Bhattacharjee M. Peripartum Diastasis of the Symphysis Pubis. N Engl J Med. 2009;361:1886.

3. Nitsche JF, Howell T. Peripartum pubic symphysis separation: a case report and review of the literature. Obstet Gynecol Surv. 2011;66:153-8.

4. Jain N, Sternberg LB. Symphyseal Separation. Obstet Gynecol. 2005;105:1229-32.

5. Idrees A. Management of chronic symphysis pubis pain following child birth with spinal cord stimulator. J Pak Med Assoc. 2012;62:71-3. 